\title{
Encerrado a oscuras: significado de vivir con esquizofrenia para diagnosticados y sus cuidadores,
Medellín-Colombia
}

\section{RESUMEN}

Vivir con esquizofrenia representa un enigma para todas aquellas personas que no presentan la enfermedad o conviven con alguien que la padece. Objetivo: comprender el significado de vivir con esquizofrenia para personas que presentan la enfermedad y sus familiares, quienes acudieron a una institución estatal para la atención de problemas de salud mental de alta complejidad en Colombia durante el 2014 y 2015. Materiales y método: se utilizó un enfoque cualitativo y la información fue analizada por medio del método de la teoría fundamentada. Las técnicas de recolección de información fueron ocho entrevistas a profundidad y dos grupos focales, con una participación de 21 personas diagnosticadas y sus cuidadores. Resultados: por medio de la codificación, el muestreo teórico, la comparación constante y los memos analíticos, se llegó a la categoría "Vivir con esquizofrenia es vivir encerrado, a oscuras y con los sueños truncados". Conclusiones: para los pacientes, la familia y para los cuidadores, vivir con esquizofrenia conlleva una sensación de encerramiento que afecta el proyecto de vida de unos y otros.

\section{PALABRAS CLAVE}

Esquizofrenia; enfermos mentales; salud mental; enfermería psiquiátrica; teoría fundamentada (Fonte: DeCS, BIREME).

\section{DOI: 10.5294/aqui.2017.17.3.7}

Para citar este artículo / To reference this article / Para citar este artigo

Zaraza-Morales DR, Hernández-Holguín DM. Encerrado a oscuras: significado de vivir con esquizofrenia para diagnosticados y sus cuidadores, Medellín, Colombia. Aquichan. 2017; 17(3): 305-315. Doi: 10.5294/aqui.2017.17.3.7

1 orcid.org/0000-0002-4197-3787. Universidad Pontificia Bolivariana, Colombia. daniel.zaraza@upb.edu.co 


\title{
Locked in Darkness: What Living with Schizophrenia Means to Diagnosed Patients and their Caregivers. Medellín - Colombia
}

\begin{abstract}
Living with schizophrenia is an enigma to all who do not have the disease or live with someone who does. Objective: The purpose of the study was to understand what living with schizophrenia means for people with the disease and their relatives, specifically in the case of patients who sought help during 2014 and 2015 at a state institution in Colombia that is dedicated to handling highly complex mental health problems. Materials and method: A qualitative approach was employed and the grounded theory methodology was used to analyze the information, which was gathered through eight in-depth interviews and two focus groups. Twenty-one individuals took part, including xx diagnosed with schizophrenia and their caregivers. Results: Through coding, theoretical sampling, constant comparison and analytical memos, a category was established; namely, "Living with schizophrenia is living locked up and in the dark, with curtailed dreams". Conclusions: For patients, their families and caregivers, living with schizophrenia leads to a sense of confinement that affects all their lives.
\end{abstract}

\section{KEYWORDS}

Schizophrenia; the mentally ill; mental health; psychiatric nursing; grounded theory (Source: DeCS, BIREME). 


\section{Envolto na escuridão: significado de viver com esquizofrenia para os diagnosticados e seus cuidadores, Medellín, Colômbia}

\section{RESUMO}

Viver com esquizofrenia representa um enigma para todas aquelas pessoas que não apresentam a doença ou convivem com alguém que a padece. Objetivo: compreender o significado de viver com esquizofrenia para pessoas que apresentam a doença e seus familiares, que recorriam a uma instituição estatal para 0 atendimento de problemas de saúde mental de alta complexidade na Colômbia durante 2014 e 2015. Métodos: foi utilizada uma abordagem qualitativa, e a informação foi analisada por meio do método da teoria fundamentada. As técnicas de coleta de informação foram oito entrevistas a profundidade e dois grupos focais, com a participação de 21 pessoas diagnosticadas e seus cuidadores. Resultados: por meio da codificação, da amostragem teórica, da comparação constante e dos relatórios analíticos, chegou-se à categoria Viver com esquizofrenia é viver preso, às escuras, e com os sonhos interrompidos. Conclusões: para os pacientes, para a família e para os cuidadores, viver com esquizofrenia implica uma sensação de encerramento que afeta o projeto de vida de todos.

\section{PALAVRAS-CHAVE}

Doentes mentais; enfermagem psiquiátrica; esquizofrenia; saúde mental; teoria fundamentada (Fuente: DeCS, BIREME). 


\section{Introducción}

La esquizofrenia es una enfermedad mental que representa un reto para los sistemas de salud en el mundo, no solo por el grado de cronicidad que conlleva, sino también por el temprano inicio de los síntomas, que afectan en su mayoría a adolescentes 0 adultos jóvenes $(1,2)$. Se estima que la prevalencia de personas con esquizofrenia es del $1 \%$ a nivel mundial (3). Dentro de las situaciones que enfrentan quienes son diagnosticados se encuentran el rechazo social, la muerte prematura por enfermedades asociadas, y la falta de tratamiento y atención oportuna, tanto así que muchos no son atendidos sino mucho tiempo después del inicio de los síntomas, y solo cuando se presenta una crisis $(4,5)$.

Se ha establecido que existen factores que pueden incrementar el riesgo de adquirirla, como es el caso del consumo de sustancias psicoactivas (6), situaciones de mucho estrés en algún momento de la vida, y la que más ha adquirido relevancia, la herencia familiar y su correspondiente carga genética $(7,8)$.

En la actualidad, se ha adelantado mucho en materia de investigación en personas con enfermedades mentales; sin embargo, la mayoría de estudios se han realizado en ambientes institucionalizados, que si bien son importantes para determinar intervenciones sobre el individuo, no permiten explorar a ciencia cierta la interacción de este con la comunidad, sus familias y lo que conlleva vivir con esquizofrenia fuera de una clínica u hospital (4, 9). Además, pocos estudios dan cuenta del significado que tiene la esquizofrenia para quienes la padecen, buscando comprender la subjetividad que viene con la experiencia de vivir y convivir con la enfermedad $(10,11)$.

Experiencias investigativas realizadas en Europa y Asia, desde un enfoque subjetivo, han mostrado la complejidad con relación al cuidado y el estigma que representa para una persona el vivir con una enfermedad como la esquizofrenia $(12,13)$. En Latinoamérica, se ha estudiado la relación entre la carga de enfermedades mentales crónicas y su impacto en la calidad de vida de las familias con las que conviven, no solo por los múltiples factores que intervienen, sino por las implicaciones culturales que permiten relacionarse positiva o negativamente con la persona cuidada (14). Elementos como la esperanza, la espiritualidad y la fuerza de voluntad se han convertido en las mejores herramientas para alcanzar la recuperación, donde el cuidado tanto de la familia como del personal de salud es fundamental $(15,16)$.
El avance en la comprensión de la percepción sobre vivir con esquizofrenia, y lo que esto conlleva, permite humanizar cada intervención por parte del profesional de enfermería (17), ya que puede sensibilizar la práctica y la gestión del cuidado, y es importante para evidenciar la forma en la que se concibe y se lleva a cabo la atención, visualizando con ello mecanismos para innovar las acciones del personal de salud en pro de la mejora de la calidad de vida de las personas diagnosticadas (18).

\section{Materiales y método}

Esta investigación tuvo un enfoque cualitativo y fue sustentada en el interaccionismo simbólico (19). La teoría fundamentada desde la propuesta de Strauss y Corbin fue el método que guió el análisis de los datos (20).

Los participantes en el estudio fueron personas diagnosticadas con esquizofrenia y sus cuidadores, residentes en el departamento de Antioquia (Colombia), que en el momento recibieran servicios y atención ambulatoria psiquiátrica. Se utilizó el muestreo teórico para la selección de los participantes (21). Se estableció un contacto directo con cada uno de ellos ya fuera en consulta externa 0 en los grupos psicoeducativos. Se realizaron ocho entrevistas individuales y dos grupos focales, de los cuales un grupo focal se desarrolló con personas que presentaban la enfermedad y otro con familiares cuidadores, para una participación en total de 21 personas. La información fue recolectada en dos tipos de escenarios, el hospitalario y el comunitario en las residencias de las personas entrevistadas. Los criterios para participar fueron los siguientes: personas mayores de edad, que hubieran sido diagnosticadas con esquizofrenia según los criterios del DSM o el CIE, y que para el momento de la entrevista estuvieran en tratamiento psiquiátrico ambulatorio; familiares de personas que hubieran sido diagnosticadas con esquizofrenia y fueran cuidadores directos de quien presentaba la enfermedad. En la tabla 1 se muestran las características de los participantes del estudio. Para asegurar la calidad de las entrevistas las guías propendieron por preguntas abiertas que fueron discutidas, además de la capacitación del investigador responsable de estas y de la revisión y los ajustes a las primeras entrevistas.

Por medio de la codificación abierta de las primeras entrevistas realizadas a los cuidadores se llegó a categorías descriptivas como autopercepción, ciclo vital, actividades ocupacionales, cuidador, familia, salud-enfermedad. A partir de un segundo grupo de entrevistas y de un análisis centrado en la codificación axial 
Tabla 1. Características de los participantes

\begin{tabular}{|c|c|c|c|c|}
\hline \multicolumn{5}{|c|}{ Personas con esquizofrenia } \\
\hline \multirow{2}{*}{ Técnica } & \multicolumn{4}{|c|}{ Participante } \\
\cline { 2 - 5 } & Código & Rol & Edad & Sexo \\
\hline EI & EP2 & Hijo & 29 & Hombre \\
\hline EI & EP4 & Hija & 20 & Mujer \\
\hline EI & EP5 & Hijo & 27 & Hombre \\
\hline EI & EP8 & Madre & 63 & Mujer \\
\hline GF & GFP9 & Hijo & 31 & Hombre \\
\hline GF & GFP10 & Padre & 64 & Hombre \\
\hline GF & GFP11 & Hijo & 39 & Hombre \\
\hline GF & GFP12 & Hijo & 59 & Hombre \\
\hline GF & GFP13 & Hijo & 26 & Hombre \\
\hline GF & GFP14 & Hijo & 31 & Hombre \\
\hline GF & GFP15 & Hijo & 30 & Hombre \\
\hline GF & GFP16 & Hijo & 49 & Hombre \\
\hline GF & GFP17 & Hijo & 48 & Hombre \\
\hline
\end{tabular}

\begin{tabular}{|c|c|c|c|c|}
\hline \multicolumn{5}{|c|}{ Familiares } \\
\hline \multirow{2}{*}{ Técnica } & \multicolumn{4}{|c|}{ Participante } \\
\cline { 2 - 5 } & Código & Rol & Edad & Sexo \\
\hline EI & EF1 & Hermana & 49 & Mujer \\
\hline EI & EF3 & Madre & 51 & Mujer \\
\hline EI & EF6 & Madre & 52 & Mujer \\
\hline GF & GFF18 & Madre & 52 & Mujer \\
\hline GF & GFF19 & Madre & 47 & Mujer \\
\hline GF & GFF20 & Madre & 56 & Mujer \\
\hline GF & GFF21 & Hermana & 49 & Mujer \\
\hline
\end{tabular}

EI: Entrevista Individual - GF: Grupo Focal

Fuente: elaboración propia.

se obtuvieron las categorías: "estar encerrado y a oscuras", "un sueño no realizado", "apoyos fragmentados" y "fortaleciendo el interior", en cada una de ellas fue posible describir a quiénes se referían los participantes, a qué hacían alusión y bajo qué circunstancias, lo que permitió avanzar en la contrastación de las nuevas categorías con las entrevistas finales, y un grupo focal con personas con esquizofrenia, para validar los hallazgos parciales e integrar las categorías en dos, interpretativas mas no saturadas: "vivir con esquizofrenia es vivir encerrado, a oscuras y con los sueños truncados" y "el valor de los apoyos, un punto subjetivo en la experiencia de vivir con esquizofrenia" (16).

Finalmente, se realizó un grupo focal con cuidadores en el que se validaron ambas categorías y la integración de "estar encerrado y a oscuras" y "un sueño no realizado", en: "vivir con esquizofrenia es vivir encerrado, a oscuras y con los sueños truncados", que es presentada en este artículo.

Durante todo el proceso de recolección y análisis hubo revisión y discusión de los investigadores, con apoyo de memos analíticos, mapas mentales y socialización con profesionales en enfermería y salud mental. Los hallazgos finales fueron socializados con personal de salud del hospital y con un grupo de cuidadores de personas diagnosticadas con esquizofrenia de la ciudad de Medellín.

Esta investigación fue formulada bajo las directrices de la Declaración de Helsinki y la Resolución 8430 de 1993 del Ministerio de Salud de Colombia. Recibió el aval ético de las instituciones participantes. Para garantizar la confidencialidad, los participantes de la investigación fueron identificados a lo largo del texto por la letra "E" (entrevistado) o "GF" (grupo focal), seguida del número correspondiente al orden de la realización de la participación (p1, p2, p3... p21).

\section{Resultados}

A continuación se presentan los hallazgos con respecto a la manera como las personas que viven con esquizofrenia y sus familias han experimentado el proceso salud-enfermedad, antes y 
después de su diagnóstico, cómo se han ido desarrollando, y los sentimientos y las emociones que les ha generado este proceso. En la siguiente categoría interpretativa se enuncia el surgimiento y desarrollo de la enfermedad en los aspectos relevantes para los participantes, y se hace énfasis en la experiencia de las crisis y la forma como aportan al aislamiento, el rechazo social y algunas experiencias de atención en los servicios de salud.

\section{Vivir con esquizofrenia es vivir encerrado, a oscuras $y$ con los sueños truncados}

El inicio de los síntomas, aunque varía en su momento y en su forma de aparecer, progresiva o repentina, es expresado como una experiencia dolorosa debido a los cambios que conlleva para la vida de la persona que es diagnosticada y su grupo familiar.

El cambio de comportamiento, que se manifiesta con mayor fuerza en el encerramiento, el temor y las reacciones extrañas para quienes los rodean, es la señal de un sufrimiento que recién empieza, no solamente para quien presenta los síntomas sino para su familia. Los cuidadores entrevistados tienen muy presentes las manifestaciones que han marcado este inicio, entre ellas, dificultades en la socialización, alucinaciones y miedo, como puede verse a continuación:

Porque es que los niños, supongamos ella... a la edad que ella tenía, pequeños, póngale usted diez, ocho, once años, les gusta mucho salir a jugar, estar juntos, estar... pues, todos unidos jugando, y ella de un momento a otro, ella no quiso volver a salir, ya se quedó, es que me acuerdo como si fuera hoy, a los once años ella se quedó encerrada y ella no volvió a salir. EF6

Era normal, él era normal, pero el año pasado él se puso agresivo y casi mata al hermanito y al papá... para mí él comenzó vomitando y al otro día ya comenzó raro y mire hasta donde llegó, a defecarse en la ropa, a hacer las necesidades en la ropa y todo... EP5

Los nuevos comportamientos extraños se relacionan con el aislamiento social, que refieren como una experiencia permanente, y las reacciones ante alucinaciones que generan temor en la persona con esquizofrenia, lo que puede derivar en reacciones agresivas y poner en peligro al grupo familiar.

Sin embargo, es la primera crisis, precedida o no por los síntomas que ya estaban alterando la tranquilidad personal y familiar, una experiencia que marca de manera más cruda el principio de la enfermedad, que abruptamente interrumpe, señala, fragmenta y divide en un antes y un después la experiencia vital. Este primer momento crítico está cargado de sensaciones y emociones como desesperación, temor y frustración, no solo en la persona enferma, sino en su familia y las personas que lo rodean. Al fragmentar la relación con el familiar, la crisis deja ver de manera más clara la manifestación de la enfermedad y el profundo sufrimiento del ser querido que la padece.

...él empezó como a alucinar, que nos iban a matar, que no podíamos salir a la calle, le cogió miedo a la policía... era agresivo... que ahí fue donde me lo remitieron para acá [institución de salud]. GFF20

Estaba empelota [desnudo] en la calle, ya cuando llegó le conté a mi esposo y entonces llamamos a la policía, y se lo llevaron para la unidad intermedia [de salud], y de allí lo remitieron al mental [institución de salud]. EF7

Estas crisis son situaciones que conllevan afectación emocional, dolor y temor en quienes las presencian, no solo por el riesgo de perder la vida sino por la impotencia de no saber cómo reaccionar ante las complejas manifestaciones de su familiar.

Las crisis que uno tiene a veces son duras porque uno se desespera, uno pasa al lado de la familia y son... no sé, es muy duro porque uno es temblando... entonces a veces a uno se le va la paloma [pierde el contacto con la realidad] y no sabe tomarse la medicación, entonces eso es muy duro porque a veces uno no consigue pensar bien o piensa solo en violencia, entonces eso es muy duro porque perjudica a la familia, también a uno. EP2

Yo, cuando él está en una crisis así, le hablo de muy lejitos porque si estoy muy cerca creo que me va a dar [golpear] con lo que tenga en la mano. EF3

Por su parte, el personal de salud, a pesar de ser una ayuda, genera sentimientos de temor y desesperanza, tanto en la persona con esquizofrenia como en sus familiares, no solo por el trauma que produce una mala atención por intervenciones insensibles con la persona que presenta la enfermedad y su familiar, sino también porque al depositar su confianza en los profesionales se espera que sus actuaciones sean en pro del bienestar físico y psicológico, lo que se torna en una contradicción cuando se violenta su integridad. 
[Una cuidadora con respecto a su familiar]...Ella ya salió, pero salió muy nerviosa, ella salió con mucho trauma, muchísimo. EF6

...Yo sí veo mucha frialdad en ellos, o sea, son muy secos, muy secos... yo a veces veo que los doctores son muy fríos, como muy secos, eso duele, eso da tristeza... falta como más entrega a los pacientes, más amor. EF7

A partir del inicio de los síntomas, cuya gravedad es ratificada con la primera crisis, comienzan en la cotidianidad de la familia una serie de explicaciones propias sobre la causa de la enfermedad. Estas explicaciones se relacionan con las creencias populares del origen de esta, como efectos de algún tipo de hechizo, posesión de demonios o el resultado entre la interacción del contexto con todos sus factores de riesgo, como el consumo de marihuana y la presión social.

...Yo decía: Ah! eso fue lo que lo puso así, la marihuana, mire ese cerebro es débil, lo puso así tanta marihuana, y de tanto pensar, y los pelaos [muchachos en la calle] en vez de ayudarlo lo desorientaron, molestándolo, tocándolo, ¿sí o no?, cuando uno el cerebro es débil y otra persona encima de uno, uno se descontrola mentalmente ¿o no? EF7

La enfermedad misma, y las experiencias que se derivan de ella, entre las cuales se encuentra la hospitalización, llevan a un aislamiento del resto de la sociedad cada vez más definitivo, y se constituye en una especie de encerramiento. Esto es descrito como una situación muy difícil, un contexto que se desconoce, y, sobre todo, un escenario que entra en choque con los ideales de la persona que sufre los síntomas de la enfermedad.

....uno estar encerrado un mes aquí no es cualquier cosa [hospital psiquiátrico], eso es algo muy duro... porque usted tiene que empezar con su subconsciente: yo no estoy loco, yo no estoy loco, yo estoy enfermo, tengo una enfermedad pero no estoy loco, pero de todas maneras usted entiende que está en un hospital psiquiátrico, pero es muy duro, pues, para mí ha sido muy duro. GFP12

Durante la primera hospitalización se genera soledad, temor e incertidumbre por desconocer lo que sucede. Este desconocimiento puede causar además desesperación, desesperanza, ansiedad y, en algunos casos, pérdida del principio de realidad, que se genera por vivir una situación nueva, lejos de la familia y de personas cercanas. "Entrevistador [con respecto a una crisis]: ¿en algún momento llegaste a sentir miedo? Participante: sí, me sentía muy solo, no sabía cómo era la movida, la salida, las luces, me encandelillaban las luces y no dormía...." EP5

A partir de las crisis y de la hospitalización surge, entonces, la idea de estar encerrado en un cuarto oscuro, donde se presentan diferentes vivencias en la experiencia de las personas que tienen la enfermedad, caracterizadas por el encerramiento y la oscuridad, en un continuo cuyo caso extremo se nombra como "descender a los infiernos".

...el hecho es que el cuarto oscuro es una forma de saber cuán oscura es la esquizofrenia cada vez que yo me sumo en mi mente... Salir de esos momentos de oscuridad, es cómo continuar viviendo y compartiendo. GFP14

...cuando uno está en el cuarto oscuro, uno baja como en un ascensor y baja y baja, y son días bajando, uno no se acuerda de comida, no se acuerda de nada,... así es la esquizofrenia. GFP10

... describir ese momento... como un lugar en el infierno, sumido en las profundidades horribles. GFP17

El diario vivir de una persona que es diagnosticada con esquizofrenia se convierte en un descender continuo emocionalmente y un ascenso de los síntomas, y a medida que lo hacen vienen las dificultades. Entre estas se encuentran los problemas para conciliar el sueño, la fluctuación emocional y los cambios nutricionales derivados de la sintomatología.

...la enfermedad va... no se queda ahí, en un solo sitio, entonces va subiendo... no dormía, porque lo que más le ha atormentado a ella es el no dormir, más que todo, y de pronto... ella es muy sentimental y de pronto a veces llora por todo. EF6

...ya no me recibía de comer porque lo iba a envenenar, si eran frijoles porque él quería lentejas, siempre era lo contrario... Además él ya no estudiaba. EP5

Para las familias es complejo saber cómo se vive dentro de ese cuarto oscuro, primero porque no se conocen los pensamientos del familiar enfermo; segundo, por la dificultad que existe para dialogar sobre la enfermedad y sus síntomas.

...nadie puede pensar como piensa otro, todos tenemos un pensamiento y es así, o sea, nadie puede meterse en el pensamiento 
del otro, qué tal que así fuera o que bueno para estas personas enfermas, sería maravilloso, por ejemplo que uno pudiera ingresar a la mente de un esquizofrénico a descubrir por qué es así. EF3

Como se puede observar, desde los cambios insinuados de comportamiento, pasando por la hospitalización y después de ella, se vive con la sensación, que a la vez se experimenta de manera física, de estar encerrado, lo cual no se relaciona solo con la falta de libertad impuesta por otras personas, sino con las consecuencias que trae el inicio de la enfermedad, con sus síntomas y las respuestas comportamentales que presenta la persona durante su cotidianidad.

Pero es un cambio muy bravo uno estar como está ahora, uno enfermo ya uno no sale, más bien como encerrado, pues, uno llega por ejemplo de aquí a la casa de uno, a uno no le provoca salir... es muy duro estar usted encerrado, no porque lo quieran mantener encerrado, sino porque a usted le nace estar encerrado, y siente a veces esa necesidad de salir de la casa, y uno sale un momentico y ahí mismo otra vez pa' dentro. GFP12

En el plano emocional la experiencia es coherente con el alejamiento social, en ella la tristeza hace presencia y de allí que la alegría es concebida como algo momentáneo, algo que se busca pero que es muy difícil que aparezca a pesar de los esfuerzos de los familiares. En contraste, se encuentra que la tristeza puede estar presente a pesar del tratamiento farmacológico prescrito para evitarla.

Entrevistador: ¿cómo es eso de vivir con esquizofrenia? Participante: eso es triste, por ejemplo yo me tomo las pastillas y no me provoca levantarme, o si uno se levanta, se levanta con mucha tristeza... La alegría le dura a uno por ahí tres minutos en el día, de resto es tristeza. GFP10

Las expectativas que se tenían sobre el proyecto de vida ahora entran en choque con la realidad dada por la vivencia de la enfermedad. Aparece entonces la desesperanza y el deseo de no tener ese diagnóstico, de ser alguien "normal", sin síntomas que alteren su vida y sus proyectos.

...ella misma me dice a mí que ella quisiera no tener nada, que ella quisiera no sentir nada de eso, porque ella tiene muchos proyectos de vida, a ella le encantaría...ella siempre desde pequeñita dice que ella quiere ser una profesional, que ella quiere trabajar, que ella quiere hacer muchas cosas, y de pronto saber que ella tiene eso [la enfermedad] que le da impedimento. EF6
Pese a la difícil experiencia ligada a la enfermedad, la recuperación es posible. Existe una "salida del cuarto", a pesar de muchos obstáculos y oscuridad. Esta recuperación se da en un marco de incertidumbre, que se mezcla con un sentimiento de desesperanza y resignación en la familia, al pensar que la presencia de la enfermedad es algo que les tocó vivir.

Entrevistador: ¿Qué piensa sobre una posible salida?

GFF18: Pues siempre hay una salida, o de pronto ya es uno acostumbrarse a convivir así, a tratar de mejorar cada día, pero ya uno como que tiene en la cabeza que eso es lo que le tocó ya.

GFF21: Eso fue lo que nos tocó a nosotros.

GFF18: Aceptar la situación y... no tratar de verla ya así, no seguir uno que es un problema, sino aceptarlo, y tratar de mejorar cada día más ese proceso.

Esta sensación de estar encerrado, de sueños no realizados, lleva a conocer las sombras, esa percepción subjetiva de oscuridad que se mantiene en la persona diagnosticada.

\section{Discusión}

Los hallazgos de la investigación permiten reconocer aspectos ya documentados con respecto a la esquizofrenia, como el hecho de ser diagnosticado que interfiere enormemente en la tranquilidad de la persona que ha sido etiquetada (22), y trae consigo cambios en todas las esferas de su cotidianidad (23), como en la social, la laboral, familiar y afectiva $(24,25)$. Estos cambios se ven reflejados en la percepción de un antes y un después, caracterizado por la falta y el deterioro en las habilidades y capacidades propias de quien se consideraba, hasta antes del diagnóstico, una persona normal (26).

La sensación de encerramiento ha sido documentada desde la psiquiatría tradicional como una manifestación clásica de los síntomas de la enfermedad $(27,28)$. Se ha descrito como un tipo de aislamiento social resultante de la desregularización en las vías serotoninérgicas y el sistema nigroestriado-dopaminérgico, que puede producir abulia y apatía (27). En otras palabras, los cambios en la neurotransmisión y la serotonina, específicamente, incrementan la sensación de estar y permanecer encerrado. Desde la neuropsicología también se ha argumentado la deficiencia 
neuronal que imposibilita las relaciones sociales en la persona que tiene esquizofrenia (29).

Una de las situaciones que genera mayor inquietud y miedo son las crisis generadas durante el proceso salud-enfermedad. Estas crisis son las manifestaciones más visibles de la heterogeneidad de los síntomas, y muestran la particularidad que existe en sus posibles etiologías y la forma de tratarlas (30). Se ha documentado que las intervenciones no adecuadas por parte del personal de salud durante un momento de crisis ponen en riesgo la vida y la recuperación de quien vive con la enfermedad o convive con alguien que la presenta (31). Esto se debe al manejo brusco y agresivo resultante de la contención física y, en otros casos, al manejo farmacológico y sus efectos adversos (26). A su vez, estas crisis incrementan la carga de cuidados que deben sobrellevar los familiares, ya que tienen que estar más pendientes de riesgos como las autolesiones o la agresión hacia otros (32).

Diferentes estudios han mostrado cómo el hecho de ser diagnosticado con una enfermedad mental acarrea una serie de consecuencias desfavorables, no solo para la persona que tiene la enfermedad y su familia $(8,33)$, sino a su vez para la comunidad, dados los altos recursos que deben destinarse para tratar la enfermedad, con el agravante de la pérdida social que se genera, pues en un gran porcentaje los síntomas inician en jóvenes en etapa productiva (34). La sensación de descenso a los infiernos también ha sido descrita por personas que han sido diagnosticadas con esquizofrenia, como un aspecto que muestra lo oscuro y aterrador que puede llegar a ser la vivencia de la enfermedad (26).

El proceso de hospitalización influye en la percepción negativa de la experiencia vivida (9). Este impacto, como lo vemos en los resultados, se asocia con el hecho de estar lejos de la familia y, por tanto, enfrentarse a un contexto que le es ajeno, lo que incrementa esa sensación de encerramiento (35). Sin embargo, como es explicado por los participantes, la recuperación es posible y esta recuperación está ligada a la percepción de esperanza y mejoramiento que tienen durante su tratamiento, que es apoyado por diferentes elementos que permiten acrecentar esa sensación $(31,36)$.

La utilización de teorías de mediano rango, como el Modelo de Recuperación de la Salud Mental, propuesto por Phil Barker, permite conceptualizar de forma amplia la importancia de introyectar aspectos del ser humano como alguien espiritual, que a través de la esperanza en su proceso de recuperación, puede trascender y ayudar a otros en su camino hacia el bienestar (37).

\section{Conclusiones}

Las ideas de desesperanza surgidas luego de no realizar ese sueño que se tenía visualizado influyen tanto en la persona que vive con la enfermedad como en su familia, y es la frustración de los sueños una experiencia dolorosa y triste. Creemos es necesario seguir realizando investigaciones que aborden la experiencia de vivir con una enfermedad mental como la esquizofrenia, desde la subjetividad de quienes son diagnosticados y sus cuidadores. Más allá de las causas etiológicas de orden fisiológico que influyan en esa sensación de encerramiento, es preciso abordar el sufrimiento, el desconocimiento y la intranquilidad que pueden gestarse también en sus familiares, quienes corren generalmente con la responsabilidad de cuidarlos. Esto, sin lugar a dudas, enriquece la forma en que se concibe la salud mental y se dan nuevos lineamientos para la formación de los profesionales de enfermería con criterios éticos y humanizados.

Conflicto de intereses: ninguno declarado.

\section{Referencias}

1. Pagsberg AK. Schizophrenia spectrum and other psychotic disorders. Eur Child Adolesc Psychiatry. 2013;22(1):S3-9.

2. Tandon R. Schizophrenia and other Psychotic Disorders in DSM-5 : Clinical implications of revisions from DSM-IV. Schizophr Relat Psychoses. 2013;16-20.

3. Organización Mundial de la Salud (OMS). The global burden of disease 2004. Geneva: OMS; 2004.

4. Millier A, Schmidt U, Angermeyer MC, Chauhan D, Murthy V, Toumi M, et al. Humanistic burden in schizophrenia: A literature review. J Psychiatr Res. 2014;54:85-93. 
5. Zaraza-Morales DR, Hernandez-Holguin DM. Towards a community mental health care for people with schizophrenia in Colombia. Cienc e Saude Coletiva. 2016;21(8).

6. Lubman DI, King JA, Castle DJ. Treating comorbid substance use disorders in schizophrenia. Int Rev Psychiatry. 2010;22(2):191-201.

7. Jablensky A. Epidemiology of schizophrenia: The global burden of disease and disability. Eur Arch Psychiatry Clin Neurosci. 2000;250:274-85.

8. Zahid MA, Ohaeri JU. Clinical and psychosocial factors associated with needs for care: an Arab experience with a sample of treated community-dwelling persons with schizophrenia. Soc Psychiatry Psychiatr Epidemiol. 2013;48(2):313-23.

9. Harvey PD, Loewenstein DA, Czaja SJ. Hospitalization and psychosis: influences on the course of cognition and everyday functioning in people with schizophrenia. Neurobiol Dis. 2013;53:18-25.

10. Manuel J, Montes G, Almería U De. Reivindicación de la persona en la esquizofrenia. Rev Int Psicol Clínica y la Salud. 2003;3:107-22.

11. Wagner LC, Geidel AR, Torres-gonzález F, King MB. Cuidado en salud mental: percepción de personas con esquizofrenia y sus cuidadores. Cienc e Saude Coletiva. 2011;16:2077-87.

12. Barut JK, Dietrich MS, Zanoni P a., Ridner SH. Sense of belonging and hope in the lives of persons with schizophrenia. Arch Psychiatr Nurs. 2015;30(2):178-84.

13. Awad G. Calidad de vida en esquizofrenia. Conceptos y medidas para la práctica clínica. Urug Rev Psiquiatr. 2012;72(2):121-9.

14. Minoletti A, Galea S. Community Mental Health Services in Latin America for people with severe mental disorders. Public Health Rev. 2014;34(2):1-23.

15. Ho RTH, Chan CKP, Lo PHY, Wong PH, Chan CLW, Leung PPY, et al. Understandings of spirituality and its role in illness recovery in persons with schizophrenia and mental-health professionals: A qualitative study. BMC Psychiatry. 2016;16(1):86.

16. Zaraza Morales DR, Hernandez Holguin DM. The value of supports for subjective well-being to live with schizophrenia. Index Enferm. 2016;25(3).

17. Sercu C, Ayala R a, Bracke P. How does stigma influence mental health nursing identities? An ethnographic study of the meaning of stigma for nursing role identities in two Belgian Psychiatric Hospitals. Int J Nurs Stud. 2015;52(1):307-16.

18. Hunter L, Weber T, Shattell M, Harris BA. Nursing students' attitudes about psychiatric mental health nursing. Issues Ment Health Nurs. 2015;36(1).

19. Milliken PJ, Schreiber R. Examining the nexus between grounded theory and symbolic interactionism. Int J Qual Methods. 2012;11(5):684-96.

20. Strauss A, Corbin J. Bases de la investigación cualitativa: técnicas y procedimientos para desarrollar la teoría fundamentada. Medellín: Antioquia U; 2002. p. 341.

21. Licqurish S, Seibold C. Applying a contemporary grounded theory methodology. Nurse Res. 2011;18(4):11-6.

22. Li J, Guo Y-B, Huang Y-G, Liu J-W, Chen W, Zhang X-Y, et al. Stigma and discrimination experienced by people with schizophrenia living in the community in Guangzhou, China. Psychiatry Res. 2017;255(36):225-31.

23. Llerena K, Reddy LF, Kern RS. The role of experiential and expressive negative symptoms on job obtainment and work outcome in individuals with schizophrenia. Schizophr Res. 2017;1-6.

24. Restrepo MU, Mora OL, Cristina ANA, Rodríguez C. Voces del estigma, Percepción de estigma en pacientes y familias con enfermedad mental. Univ Médica. 2007;48:207-20.

25. Silva C, Texeira W, Bandeira M, Siqueira A, Silva M, Penido J. Qualidade de vida e dimensão ocupacional na esquizofrenia: uma comparação por sexo Quality of life and occupational domain in schizophrenia: a gender comparison. Cad SaudePública, Rio Janeiro. 2006;22(6):1303-14. 
26. Noiseux S, Ricard N. Recovery as perceived by people with schizophrenia, family members and health professionals: A grounded theory. Int J Nurs Stud. 2008;45(8):1148-62.

27. Jerónimo SR, Diego C. DLVS, Patricia SP. Bases neurobiológicas de la esquizofrenia. Clínica y Salud. 2010;21(3):235-54.

28. Zipursky RB, Reilly TJ, Murray RM. The myth of schizophrenia as a progressive brain disease. Schizophr Bull. 2013;39(6):1363-72.

29. Meesters PD, Comijs HC, de Haan L, Smit JH, Eikelenboom P, Beekman ATF, et al. Subjective quality of life and its determinants in a catchment area based population of elderly schizophrenia patients. Schizophr Res; 2013;147(2-3):275-80.

30. Li JB, Liu WI, Huang MW. Integrating evidence-based community-care services to improve schizophrenia outcomes: A preliminary trial. Arch Psychiatr Nurs. 2015;30(1):102-8.

31. McEvoy P, Schauman O, Mansell W, Morris L. The experience of recovery from the perspective of people with common mental health problems: findings from a telephone survey. Int J Nurs Stud. 2012;49(11):1375-82.

32. Hanzawa S, Bae J-K, Bae YJ, Chae M-H, Tanaka H, Nakane H, et al. Psychological impact on caregivers traumatized by the violent behavior of a family member with schizophrenia. Asian J Psychiatr; 2013;6(1):46-51.

33. Kate N, Grover S, Kulhara P, Nehra R. Relationship of caregiver burden with coping strategies, social support, psychological morbidity, and quality of life in the caregivers of schizophrenia. Asian J Psychiatr. 2013;6(5):380-8.

34. Organización Mundial de la Salud (OMS). Plan de acción sobre salud mental 2013-2020. Geneva: OMS; 2013.

35. Graor CH, Knapik GP. Addressing methodological and ethical challenges of qualitative health research on persons with schizophrenia and bipolar disorder. Arch Psychiatr Nurs. 2013;27(2):65-71.

36. Kelly M, Gamble C. Exploring the concept of recovery in schizophrenia. J Psychiatr Ment Health Nurs. 2005;12(2):245-51.

37. Barker P, Buchanan-Barker P. The tidal model of mental health recovery and reclamation: application in acute care settings. Issues Ment Health Nurs. 2010;31(3):171-80. 